\title{
Targeting the cancer lesion, not the whole prostate
}

\author{
Nishant Bedi ${ }^{1,2}$, Deepika Reddy ${ }^{1,2}$, Hashim U. Ahmed ${ }^{1,2}$ \\ ${ }^{1}$ Imperial Urology, Charing Cross Hospital, Imperial College Healthcare NHS Trust, London, UK; ${ }^{2}$ Imperial Prostate, Division of Surgery, \\ Department of Surgery and Cancer, Imperial College London, London, UK \\ Contributions: (I) Conception and design: N Bedi, HU Ahmed; (II) Administrative support: None; (III) Provision of study materials or patients: None; \\ (IV) Collection and assembly of data: N Bedi; (V) Data analysis and interpretation: N Bedi; (VI) Manuscript writing: All authors; (VII) Final approval \\ of manuscript: All authors. \\ Correspondence to: Nishant Bedi. Urology Department, Charing Cross Hospital, Imperial College NHS Trust, London, UK. Email: nishantbedi@nhs.net.
}

\begin{abstract}
Modern cancer treatment aims to conserve as much healthy tissue as possible. This has been challenging in the treatment of prostate cancer due to the difficulty in imaging the gland and concerns over leaving multifocal cancer untreated. With improvements in imaging and understanding of multifocal prostate cancer evidence now shows accurate treatment of just the primary focus of cancer or the index lesion can control progression or recurrence of the disease. Many different energy sources are now available to target the cancer lesion within the prostate with less significant side-effects on urinary and sexual function compared to radical treatment. Evidence shows that men value these functions highly and would even trade years of life in exchange for preserved retention of continence or erectile function. Focal treatment of prostate cancer aims to provide both cancer control and preservation of sexual and urinary functions so that men do not have to make a choice between the two. This is a treatment option that men clearly want and deserve.
\end{abstract}

Keywords: Prostate cancer; focal therapy; high-intensity focused ultrasound (HIFU); cryotherapy; index lesion; irreversible electroporation (IRE); vascular-targeted photodynamic therapy (VTP); focal brachytherapy; laser interstitial therapy (LITT); focal radiofrequency ablation (focal RFA); photodynamic therapy (PDT)

Submitted Aug 12, 2019. Accepted for publication Sep 04, 2019.

doi: $10.21037 / \operatorname{tau} .2019 .09 .12$

View this article at: http://dx.doi.org/10.21037/tau.2019.09.12

\section{Introduction}

Cancer treatment once sought larger and larger tumour resections in an effort to clear the tumour and prevent recurrences. Famously Halsted pioneered a radical mastectomy in the $19^{\text {th }}$ century which involved removing the whole breast along with underlying muscles pectoralis major and minor along with the lymph nodes. This left patients with little arm movement, large granulating wounds and chronic pain. Today, minimal breast tissue resection is coupled with adjuvant treatment resulting in the same cancer control but significantly fewer side-effects. Treatment of prostate cancer has followed a similar path, as radical treatment compromises lifestyle severely by producing incontinence and erectile dysfunction. This has resulted in developments in focal therapies which seek to safely treat the cancer without compromising functional outcomes.

\section{The index lesion}

Prostate cancer is multi-focal in the majority of cases and therefore traditional treatment has focused on treating the whole gland, to avoid sparing any cancer cells (1). Within one gland both low grade and high grade or significant cancer may exist $(2,3)$. The "project to eliminate lethal prostate cancer" (PELICAN) study showed that in patients with multiple metastases these were almost always monoclonal and had therefore originated from one cell of prostate cancer in a primary tumour (4). Further evidence has indicated that usually the aggressive clone originates from one lesion, known as the index lesion, such that if 
left untreated it can lead to a lethal metastatic state (2). The secondary low-grade lesions are rarely lethal or likely to metastasize and may not require active treatment $(5,6)$. Multifocal disease is present in many other cancers, such as renal, thyroid, lung, breast and liver, in which tissuepreserving therapy is now standard (7).

Pathology studies have shown that, once the main or index lesion has been identified, it is usually accompanied by a low grade or non-significant lesion (8). When template-mapping biopsies of the prostate were performed, $46-59 \%$ of patients had one significant or index lesion and only $10-14 \%$ had more than one significant lesion (9). The $10 \%$ of cases where more than one clinically significant lesion is present should be carefully identified and offered appropriate radical treatment if possible.

The incidence of unifocal cancers in radical prostatectomy specimens is in the region of $13-38 \%$ (10). If prostate cancer is noted to be unilateral in approximately one third of men who have surgery, treating half the gland with hemi-ablation may provide suitable treatment of this population's prostate cancer (11). Improvements in diagnosis and targeted treatment mean that the part of the prostate containing the index cancer lesions can often be treated alone (12).

\section{Gleason grading, tumour volume and insignificant lesions}

In recent years the significance of Gleason grade 3 disease has been under debate, with a growing consensus that its classification might be downgraded from cancer to a benign entity (13). This would reflect the downgrading of Gleason 1 and 2 from the original scoring system, described by Donald Gleason in 1966. Gleason 1 and 2 originally were described as well-differentiated tumour cells and were included in the original scoring system as cancer. Future work showed that there was no malignant risk from either grade and therefore they are no longer used in the current Gleason scoring system $(14,15)$. Removing the term "cancer" removed an unnecessary burden on both the patient and the clinician. As the evidence grows that Gleason 3 has a similar low malignant potential then it could shortly follow suit.

If true Gleason 3 prostate cancer does not actually demonstrate any of the traditional hallmarks of cancer removing the label may have significant impact. The word "cancer" understandably causes anxiety to patients, as does their expectation of the burden of treatment. The example set by the renaming of 'grade one superficial bladder cancer' as 'papillary urothelial neoplasm of low malignancy potential' (PUNLUMP) has already shown benefit to the bladder cancer treatment pathway and suggests that Gleason 3 prostate cancer might also helpfully be renamed $(15,16)$. One suggestion from Esserman and colleagues involves removing the term cancer for "indolent lesions of epithelial origin" (IDLE) instead (17).

The Prostate Cancer Intervention Versus Observation (PIVOT) trial first demonstrated that radical treatment of pure pattern 3 did not carry a survival benefit, demonstrated now at 20 years follow-up $(18,19)$. Other studies have shown that only 1.4 men out of 1,000 with untreated Gleason $3+3$ disease would die from their disease within 30 years. However some suggest that Gleason 6 disease does not metastasize and therefore it has been argued that the Gleason 6 disease is not significant in the prostate cancer outcomes of the patient (15). Diagnosis of prostate cancer in the past has suffered inaccuracy and it is possible that some more aggressive cancer may have been missed causing understaging at initial diagnosis.

In the ProtecT trial a small number of men with Gleason 6 prostate cancers, diagnosed using sextant systematic biopsies, metastasis and prostate-cancer related deaths were observed. There is debate as to whether, in those patients, the disease was true Gleason 6, as it was identified by TRUS biopsy without MRI or targeted biopsies. We now know this diagnostic pathway has a higher likelihood of missing some pattern 4 , or even higher grade cancer, resulting in understaging of these tumours (20). These results are not borne out by other trials discussed regarding Gleason 3 disease.

The SPCG-4 study randomised men with localised prostate cancer to radical prostatectomy or to watchful waiting. Now, at 29 years follow-up, findings showed men with Gleason score of 6 had a similar low risk of death from prostate cancer to men with a Gleason score of $3+4$ (relative risk, $0.99 ; 95 \%$ CI, 0.23 to 4.33 ), but the risk with a Gleason score of $4+3$ was 5 times as high (relative risk, $5.73 ; 95 \%$ CI, 1.59 to 20.67) (21). Therefore some studies also argue that a small amount of pattern 4 disease along with that graded Gleason 3 is clinically insignificant, as it does not tend to metastasize or cause mortality.

Modern Gleason 3 disease is likely to be even less malignant than when these long term trials started following an adjustment in 2005, when the International Society of Urological Pathology (ISUP) adjusted the diagnostic criteria for Gleason 3 and 4 disease, so that more aggressive appearances were upgraded from Gleason 3 to 4 (22). 
Therefore, following this change, "Gleason 3+3" disease has had an even better outcome than previous research would have suggested; this is known as the "Will Rogers" phenomenon (23).

Therefore the evidence suggests pure pattern 3 does not carry the risk of metastasis or progression as is the case with other cancers (24); there is no clinical risk to patients. In which case overdiagnosis and overtreatment may be causing excessive cost and psychological distress to patients (15).

\section{Improvement in prostate cancer diagnostics and disease characterisation}

Prostate cancer diagnosis has undergone a revolution, as have the accepted paradigms in other areas of medicine, by adding MRI imaging prior to invasive biopsies. Although mpMRI is not a perfect detection tool, it can have sensitivity up to $93 \%$ in identifying clinically significant lesions, compared to the $48 \%$ sensitivity of TRUS biopsy alone, as shown in the PROMIS study. Allowing the authors to conclude that mpMRI triage might allow $27 \%$ of patients to avoid a primary biopsy (25). The PRECISION study has since shown the advantages of mpMRI-guided biopsies in identifying clinically significant cancer, as opposed to insignificant cancer. The MRI with targeted biopsy arm detected $38 \%$ of significant and $9 \%$ of insignificant cancers, compared to the standard of care (12-core TRUS random systematic biopsy), which detected $26 \%$ of clinically significant cancer and $22 \%$ of insignificant. Clinically significant cancer was defined as the presence of a single biopsy core indicating disease of Gleason score $3+4$ or greater (26).

The latest Cochrane review of prostate cancer detection compares the two pathways: MRI to determine biopsy or "MRI pathway" and the traditional upfront TRUS biopsy pathway or "systematic biopsy". The results show the MRI pathway to be superior as it is $12 \%$ more likely to make the correct diagnosis. Most benefit is seen in men who have had a negative biopsy, where the MRI pathway is $44 \%$ more likely to make the correct diagnosis. The authors note that the MRI pathway can still miss some clinically significant prostate cancer. Follow-up should be arranged to particularly monitor those with risk factors, such as family history, race or palpable nodules (27).

\section{Focal therapy}

Despite the best developments of robotic surgery and targeted radiotherapy, these procedures carry a risk of impotence (30-50\%), incontinence (5-20\%) and rectal toxic effects $(5-20 \%)$. A strategy that is used to treat clinically significant unilateral lesions in localised prostate cancer might result in fewer side-effects while retaining cancer control $(28,29)$.

Discrete experiments examining patients' choice of treatment have shown that patients do wish to avoid sideeffects from medical treatment when given the choice (30). In the COMPARE study men with prostate cancer were asked whether they would trade-off some survival for a reduction of side-effects on urinary function and erections. The results from 468 men with prostate cancer were on average, that patients were willing to trade $0.68 \%$ and $0.28 \%$ survival for $1 \%$ chance of improving urinary function and $1 \%$ chance of keeping erections, respectively. Thus this shows that the side-effects of incontinence and impotence with radical treatment are significant enough for most men to be prepared to lose months to years of life in return for avoiding certain functional side-effects (31).

With more accurate imaging showing exactly where the prostate cancer tumours are located within the prostate. Focal treatment of prostate cancer has become practicable. Several different energy sources have been used to deliver focal therapy to the prostate with varying benefits on cancer control and on the incidence of side-effects (32).

High-intensity focused ultrasound (HIFU) is delivered directly by a transrectal probe, which both images and delivers thermal energy to the prostate. A temperature of up to $60{ }^{\circ} \mathrm{C}$ is achieved within the prostate, in a field approximately the size of a rice grain. The energy causes a local cavitation effect which results in tissue necrosis and death. The most recent published data is from a UK based, multicentre trial including 625 consecutive patients with clinically significant prostate cancer treated with focal HIFU. At 5-year follow-up, failure-free survival, metastasisfree survival, cancer-specific survival, and overall survival were $88 \%, 98 \%, 100 \%$, and $99 \%$, respectively. Urinary incontinence (any pad use) was 2\% (33).

One French multi-institution prospective trial treated 111 patients with localized prostate cancer with HIFU hemiablation. At 1-year follow-up targeted prostate biopsies showed a $95 \%$ absence of clinically significant cancer. Radical treatment-free survival rate was $89 \%$ at 2 years. At 12 months, continence and erectile functions were preserved in $97 \%$ and $78 \%$ (34). The end-point which was clinically significant cancer on biopsy was criticized as not being clinically relevant. However, in reply the 
authors defended the use of biopsies of the treated tissue to demonstrate successful ablation and of the non-treated gland as a surrogate for progression (35).

There have been 13 previous studies evaluating focal HIFU. On average, significant adverse events occurred in 1.5\% (IQR: $0-3.2 \%$ ) of patients. Pad-free continence and potency preservation were achieved in $100 \%$ (32). The INDEX trial will be the first multi-centre, medium-term follow-up trial evaluating outcomes in men treated with HIFU for localised prostate cancer. The trial has recruited patients and results are awaited (36). The CHRONOS (Comparative Health Research Outcomes of Novel Surgery in prostate cancer) and PART randomised controlled trials are due to start recruitment shortly. These will be the first trials to evaluate progression-free-survival rates in clinically significant prostate cancer treated with either focal therapy or radical treatment (surgery or radiotherapy).

Cryotherapy works by reducing the temperature of tissue to $-40^{\circ} \mathrm{C}$ and then thawing, in at least two cycles, causing necrosis and apoptosis. Prostate cancer patients treated with cryotherapy are added to the Cryo On-Line Data (COLD) registry. A review of 300 men with high-grade, clinically localised, prostate cancer was performed using the COLD registry. The 5 -year biochemical progression-free survival [(using the Phoenix criteria (nadir $+2 \mathrm{ng} / \mathrm{mL}$ )] was $59.1 \%$. Complete continence was noted in $90.5 \%$ or men and potency in $17 \%$ at 12 -month follow-up (37). Analysis comparing whole gland cryotherapy against partial ablation in propensity score-matched pairs for intermediate-risk prostate cancer showed similar biochemical progressionfree survival, with either the Phoenix or ASTRO criteria. However, sexual function outcomes were improved with partial ablation with potency rates of $29.5 \%$ for whole gland and $46.8 \%$ for partial ablation. Retention and continence rates were similar, and rectourethral fistula rates were $1.2 \%$ and $0 \%$ respectively (38).

Comparison with radical prostatectomy has demonstrated similar oncological outcomes in one retrospective, matchedpair analysis comparing focal cryotherapy to radical prostatectomy for clinically unilateral prostate cancer, at a median 3.7-year follow-up (39). In a recent multicentre study of 122 patients undergoing focal cryotherapy for medium to high-risk prostate cancer, at 3-year follow-up, no patient died from their cancer whilst failure-free survival was approximately $90 \%$. None of the patients needed pads for managing urine leakage although $16 \%$ had erection problems. There were no rectal adverse events. These results may reflect the improvement in the delivery of cryotherapy for prostate cancer (40).

Photodynamic therapy (PDT) ablation relies on the laser activation of a vascular photosensitiser. This causes the local formation of reactive oxygen species resulting in vessel thrombosis, apoptosis and necrosis. Padeliporfin is administered intravenously and optical fibres are inserted transperineally into the prostate within the target zone and activated by laser light $753 \mathrm{~nm}$ with a fixed power of 150 $\mathrm{mW} / \mathrm{cm}$ for $22 \mathrm{~min} 15 \mathrm{~s}$. In the only currently published study, the authors controversially used active surveillance as the standard of care. The patients treated all had Gleason 3 disease, and most centres would not offer such patients any treatment. At a median 24 months followup $28 \%$ had disease progression in the treatment group compared to $58 \%$ in the active surveillance group. Note that $58 \%$ is a surprisingly high number for progression on active surveillance; although active surveillance varies greatly between centres, the 5 -year progression rate might be expected to be in the range of $14-50 \%$ (41). The most common adverse event of this treatment was prostatitis seen in 2\%; $1 \%$ (2 of 206) of patients suffered from erectile dysfunction (42). The latest NICE guidelines have not approved PDT ablation for prostate cancer treatment due to its cost and the weak evidence presented in this study (43).

VTP is vascular-targeted photodynamic therapy using TOOKAD $\left({ }^{\circledR}\right)$ soluble. The TOOKAD soluble is injected intravenously and activated by light-diffusing fibres placed transperineally. One initial study used endpoints of MRI at 1-week post procedure and prostate biopsy at 6 months. At 6 months $61 / 83(73 \%)$ of patients who underwent prostate biopsies were negative for cancer. In total 75/86 (87\%) patients suffered at least one mild or moderate adverse event and 8 (9\%) had a serious adverse event (44). In a medium term study at 3.5 years follow-up successful ablation of cancer was seen in $51 / 68(75 \%)$ patients. In cases of recurrence/persistence of malignancy the Gleason score either remained the same or rose by 1 point, i.e., to $3+4$ for eight patients and $4+3$ for two patients. There were 64 related adverse events, $48 \%$ were Clavien grade I, 47\% were grade II, and $5 \%$ were grade III (45).

Laser interstitial therapy (LITT) involves using laser fibres placed directly around a prostate lesion; in-bore or ultrasound monitoring is used to detect the tissue temperature (46). Four prospective studies evaluating focal LITT in 50 patients have been reported in the literature (32). TRUS standard and MRI were systematically used to identify eligible patients. One study included only men with low-risk disease, whereas the other studies included 
also Gleason $<8$. At early (3 months) follow-up no prostate cancer was detected in targeted biopsies and the probability of transition to secondary local treatment was $0 \%$. Padfree continence and potency preservation were achieved in $100 \%$ (47).

Focal brachytherapy, as opposed to whole gland radiotherapy, uses no external beam radiation. The radioactive seeds are placed in the targeted area of the gland via a transperineal approach (48). Two retrospective Stage $2 \mathrm{a}-\mathrm{b}$ case series evaluating focal brachytherapy have been reported in the literature $(48,49)$. The study population included low and intermediate risk patients with a median age of 62.3 years and a median of PSA of $6 \mathrm{ng} / \mathrm{mL}$. At a median 5.1 year follow up in one of the series no patient had secondary local treatment. Pad-free continence was reported only by one series and was at $95.2 \%$. Potency preservation was not reported by either series (32). A more recent study offered "ultra" focal brachytherapy to patients as an alternative to active surveillance. In total 17 patients were treated. End points were MRI and biopsy at 1 year. It was noted that MRI follow up for the treated volume was of little value due to artefact from the brachytherapy titanium seeds. No recurrence was noted in the treated volume, 7 non clinically significant cancer and one Gleason 3+4 were observed in untreated tissue. No urinary incontinence or erectile dysfunction reported (50).

Irreversible electroporation (IRE) is a non-thermal ablation technique, delivering high-voltage, low-energy, electric current within the target tissue. In the prostate, this is achieved by positioning electro-needles transperineally. One proof of concept Stage 1 and two retrospective cases series Stage 2 a studies have been reported in the literature. Patients with low to intermediate disease were treated. The probability of transition to secondary local treatment was $11.9 \%$. Overall survival was $100 \%$ at short term follow up. Pad-free continence and potency preservation were achieved in $100 \%$ (51-53).

Radiofrequency ablation (RFA) delivers medium frequency alternating current to generate heat, through needles placed transperineally. An initial proof of concept Stage 1 study, evaluating focal RFA prior to radical prostatectomy in 15 men, showed that the RFA energy delivery-system created a necrotic lesion in the prostate tissue in a reproducible and controlled manner (54). The full ProRAFT study results are awaited, using a bipolar coil design (Encage device), preliminary results showed that 20 men, with localised disease not eligible for active surveillance were treated. At 6 months repeat biopsy
$16 / 20(80 \%)$ had no significant disease or new cancer and surveillance MRI showed no progression. At 1-year functional follow-up one patient with an apical tumour had suffered urinary leakage but there was no deterioration in sexual function (55).

Focal therapy ablation patterns can target just the lesion or treat half the gland or three-quarters in a "hockey stick" shape (7). Selecting focal technologies to best target the specific lesion in a bespoke manner will allow the merits of each technology to be maximised (56). Examples may include utilising cryotherapy for anterior tumours in large prostates; this may be advantageous as the probes can be placed directly around the anterior lobe thus avoiding the longer distance required for delivering energy from the rectum. HIFU is generally more suited to peripheral tumours in smaller glands.

\section{Conclusions}

Prostate cancer can be unifocal in up to $38 \%$ of the cases in which radical treatment is performed. Treating just the primary focus of cancer or the index lesion may control progression or recurrence of the disease. Gleason 6 disease does not benefit from treatment. Modern imaging and biopsy techniques allow accurate identification of the primary focus of clinically significant cancer. Many different energy sources which can be used to attack the tumour are now available and there is a growing body of evidence to confirm that good cancer control can be achieved with less significant side-effects such as adverse actions on urinary and sexual function. Evidence shows that patients do not want side-effects from medical treatment and would even trade years of life in exchange for preserved retention of continence or erectile function. Current trials hope to confirm these promising results and establish more firmly and widely this treatment option for men who clearly want and deserve it.

\section{Acknowledgments}

Funding: None.

\section{Footnote}

Provenance and Peer Review: This article was commissioned by the Guest Editors (Martin J. Connor, Saiful Miah, Taimur T. Shah, Hashim U. Ahmed) for the series "Prostate Imaging and Focal Therapy" published in Translational 
Andrology and Urology. The article was sent for external peer review organized by the Guest Editors and the editorial office.

Conflicts of Interest: All authors have completed the ICMJE uniform disclosure form (available at http://dx.doi. org/10.21037/tau.2019.09.12). The series "Prostate Imaging and Focal Therapy" was commissioned by the editorial office without any funding or sponsorship. HUA served as an unpaid Guest Editor of the series. DR is a clinical research fellow funded by a research grant from Prostate Cancer UK. HUA reports grants and personal fees from Sonacare, grants and personal fees from Boston Scientific, grants and personal fees from Sophiris Biocorp, grants from Trod medical, NB has no other conflicts of interest to declare.

Ethical Statement: The authors are accountable for all aspects of the work in ensuring that questions related to the accuracy or integrity of any part of the work are appropriately investigated and resolved.

Open Access Statement: This is an Open Access article distributed in accordance with the Creative Commons Attribution-NonCommercial-NoDerivs 4.0 International License (CC BY-NC-ND 4.0), which permits the noncommercial replication and distribution of the article with the strict proviso that no changes or edits are made and the original work is properly cited (including links to both the formal publication through the relevant DOI and the license). See: https://creativecommons.org/licenses/by-nc-nd/4.0/.

\section{References}

1. Humphrey PA. Complete histologic serial sectioning of a prostate gland with adenocarcinoma. Am J Surg Pathol 1993;17:468-72.

2. Liu W, Laitinen $\mathrm{S}$, Khan $\mathrm{S}$, et al. Copy number analysis indicates monoclonal origin of lethal metastatic prostate cancer. Nat Med 2009; 15:559-65.

3. Karavitakis M, Ahmed HU, Abel PD, et al. Anatomically versus biologically unifocal prostate cancer: a pathological evaluation in the context of focal therapy. Ther Adv Urol 2012;4:155-60.

4. Gundem G, Van Loo P, Kremeyer B, et al. The evolutionary history of lethal metastatic prostate cancer. Nature 2015;520:353-7.

5. Klotz L. Prostate cancer overdiagnosis and overtreatment.
Curr Opin Endocrinol Diabetes Obes 2013;20:204-9.

6. Klotz L. Cancer overdiagnosis and overtreatment. Curr Opin Urol 2012;22:203-9.

7. Valerio M, Ahmed HU, Emberton M, et al. The role of focal therapy in the management of localised prostate cancer: a systematic review. Eur Urol 2014;66:732-51.

8. Bott SR, Ahmed HU, Hindley RG, et al. The index lesion and focal therapy: an analysis of the pathological characteristics of prostate cancer. BJU Int 2010;106:1607-11.

9. Valerio M, Anele C, Freeman A, et al. Identifying the index lesion with template prostate mapping biopsies. J Urol 2015;193:1185-90.

10. Eggener SE, Scardino PT, Carroll PR, et al. Focal therapy for localized prostate cancer: a critical appraisal of rationale and modalities. J Urol 2007;178:2260-7.

11. Polascik TJ, Mayes JM, Schroeck FR, et al. Patient selection for hemiablative focal therapy of prostate cancer: variables predictive of tumor unilaterality based upon radical prostatectomy. Cancer 2009;115:2104-10.

12. Mazzucchelli R, Scarpelli M, Cheng L, et al. Pathology of prostate cancer and focal therapy ('male lumpectomy'). Anticancer Res 2009;29:5155-61.

13. Lepor H, Donin NM. Gleason 6 prostate cancer: serious malignancy or toothless lion? Oncology (Williston Park) 2014;28:16-22.

14. Gleason DF. Classification of prostatic carcinomas. Cancer Chemother Rep 1966;50:125-8.

15. Miah S, Ahmed HU, Freeman A, et al. Does true Gleason pattern 3 merit its cancer descriptor? Nat Rev Urol 2016;13:541-8.

16. Ahmed HU, Arya M, Freeman A, et al. Do low-grade and low-volume prostate cancers bear the hallmarks of malignancy? Lancet Oncol 2012;13:e509-17.

17. Esserman L, Shieh Y, Thompson I. Rethinking screening for breast cancer and prostate cancer. JAMA 2009;302:1685-92.

18. Wilt TJ. The Prostate Cancer Intervention Versus Observation Trial: VA/NCI/AHRQ Cooperative Studies Program \#407 (PIVOT): design and baseline results of a randomized controlled trial comparing radical prostatectomy with watchful waiting for men with clinically localized prostate cancer. J Natl Cancer Inst Monogr 2012;2012:184-90.

19. Wilt TJ, Jones KM, Barry MJ, et al. Follow-up of Prostatectomy versus Observation for Early Prostate Cancer. N Engl J Med 2017;377:132-42.

20. Hamdy FC, Donovan JL, Lane JA, et al 10-Year Outcomes 
after Monitoring, Surgery, or Radiotherapy for Localized Prostate Cancer. N Engl J Med 2016;375:1415-24.

21. Bill-Axelson A, Holmberg L, Garmo H, et al. Radical Prostatectomy or Watchful Waiting in Prostate Cancer 29-Year Follow-up. N Engl J Med 2018;379:2319-29.

22. Epstein JI, Allsbrook WC Jr, Amin MB, et al. The 2005 International Society of Urological Pathology (ISUP) Consensus Conference on Gleason Grading of Prostatic Carcinoma. Am J Surg Pathol 2005;29:1228-42.

23. Montironi R, Cheng L, Lopez-Beltran A, et al. Original Gleason system versus 2005 ISUP modified Gleason system: the importance of indicating which system is used in the patient's pathology and clinical reports. Eur Urol 2010;58:369-73.

24. Edison E, Tariq Shah T, Ahmed HU. Focal Ablation of Early-Stage Prostate Cancer: Candidate Selection, Treatment Guidance, and Assessment of Outcome. Urol Clin North Am 2017;44:575-85.

25. Ahmed HU. The PROMIS of MRI. BJU Int 2016;118:7.

26. Kasivisvanathan V, Rannikko AS, Borghi M, et al. MRITargeted or Standard Biopsy for Prostate-Cancer Diagnosis. N Engl J Med 2018;378:1767-77.

27. Drost FH, Osses DF, Nieboer D, et al. Prostate MRI, with or without MRI-targeted biopsy, and systematic biopsy for detecting prostate cancer. Cochrane Database Syst Rev 2019;4:CD012663.

28. Bass EJ, Ahmed HU. Focal therapy in prostate cancer: A review of seven common controversies. Cancer Treat Rev 2016;51:27-34.

29. Ahmed HU. The index lesion and the origin of prostate cancer. N Engl J Med 2009;361:1704-6.

30. King MT, Hall J, Lancsar E, et al. Patient preferences for managing asthma: results from a discrete choice experiment. Health Econ 2007;16:703-17.

31. Ahmed HU WV, McCartan N, et al. Evaluating the tradeoffs men with localised prostate cancer make between the risks and benefits of treatments: The COMPARE study 2018 Available online: http://abstracts.ncri.org.uk/ abstract/evaluating-the-trade-offs-men-with-localisedprostate-cancer-make-between-the-risks-and-benefits-oftreatments-the-compare-study/

32. Valerio M, Cerantola Y, Eggener SE, et al. New and Established Technology in Focal Ablation of the Prostate: A Systematic Review. Eur Urol 2017;71:17-34.

33. Guillaumier S, Peters M, Arya M, et al. A Multicentre Study of 5-year Outcomes Following Focal Therapy in Treating Clinically Significant Nonmetastatic Prostate Cancer. Eur Urol 2018;74:422-9.
34. Rischmann P, Gelet A, Riche B, et al. Focal High Intensity Focused Ultrasound of Unilateral Localized Prostate Cancer: A Prospective Multicentric Hemiablation Study of 111 Patients. Eur Urol 2017;71:267-73.

35. Rischmann P. Reply to Thomas Zilli, Gilles Crehange and Olivier Chapet's Letter to the Editor re: Pascal Rischmann, Albert Gelet, Benjamin Riche, et al. Focal High Intensity Focused Ultrasound of Unilateral Localized Prostate Cancer: A Prospective Multicentric Hemiablation Study of 111 Patients. Eur Urol 2017;71:267-73. Eur Urol 2017;72:e15-6.

36. Dickinson L, Ahmed HU, Kirkham AP, et al. A multicentre prospective development study evaluating focal therapy using high intensity focused ultrasound for localised prostate cancer: The INDEX study. Contemp Clin Trials 2013;36:68-80.

37. Tay KJ, Polascik TJ, Elshafei A, et al. Primary Cryotherapy for High-Grade Clinically Localized Prostate Cancer: Oncologic and Functional Outcomes from the COLD Registry. J Endourol 2016;30:43-8.

38. Tay KJ, Polascik TJ, Elshafei A, et al. Propensity ScoreMatched Comparison of Partial to Whole-Gland Cryotherapy for Intermediate-Risk Prostate Cancer: An Analysis of the Cryo On-Line Data Registry Data. J Endourol 2017;31:564-71.

39. Bahn D, de Castro Abreu AL, Gill IS, et al. Focal cryotherapy for clinically unilateral, low-intermediate risk prostate cancer in 73 men with a median follow-up of 3.7 years. Eur Urol 2012;62:55-63.

40. Shah TT, Peters M, Eldred-Evans D, et al. EarlyMedium-Term Outcomes of Primary Focal Cryotherapy to Treat Nonmetastatic Clinically Significant Prostate Cancer from a Prospective Multicentre Registry. Eur Urol 2019;76:98-105.

41. Kinsella N, Helleman J, Bruinsma S, et al. Active surveillance for prostate cancer: a systematic review of contemporary worldwide practices. Transl Androl Urol 2018;7:83-97.

42. Azzouzi AR, Vincendeau S, Barret E, et al. Padeliporfin vascular-targeted photodynamic therapy versus active surveillance in men with low-risk prostate cancer (CLIN1001 PCM301): an open-label, phase 3, randomised controlled trial. Lancet Oncol 2017;18:181-91.

43. NICE. Single Technology Appraisal Padeliporfin for treating localised prostate cancer [ID866] Committee Papers 2018 [cited 2019 10/07/2019]. Available online: https://www.nice.org.uk/guidance/ta546/evidence/ appraisal-consultation-document-committee-papers- 
pdf-6599593550

44. Azzouzi AR, Barret E, Moore CM, et al. TOOKAD(®) Soluble vascular-targeted photodynamic (VTP) therapy: determination of optimal treatment conditions and assessment of effects in patients with localised prostate cancer. BJU Int 2013;112:766-74.

45. Noweski A, Roosen A, Lebdai S, et al. Medium-term Follow-up of Vascular-targeted Photodynamic Therapy of Localized Prostate Cancer Using TOOKAD Soluble WST-11 (Phase II Trials). Eur Urol Focus 2019;5:1022-8.

46. Lindner U, Weersink RA, Haider MA, et al. Image guided photothermal focal therapy for localized prostate cancer: phase I trial. J Urol 2009;182:1371-7.

47. Lepor H, Llukani E, Sperling D, et al. Complications, Recovery, and Early Functional Outcomes and Oncologic Control Following In-bore Focal Laser Ablation of Prostate Cancer. Eur Urol 2015;68:924-6.

48. Cosset JM, Cathelineau X, Wakil G, et al. Focal brachytherapy for selected low-risk prostate cancers: a pilot study. Brachytherapy 2013;12:331-7.

49. Nguyen PL, Chen MH, Zhang Y, et al. Updated results of magnetic resonance imaging guided partial prostate brachytherapy for favorable risk prostate cancer: implications for focal therapy. J Urol 2012;188:1151-6.

50. Graff P, Portalez D, Lusque A, et al. IDEAL 2a Phase

Cite this article as: Bedi N, Reddy D, Ahmed HU. Targeting the cancer lesion, not the whole prostate. Transl Androl Urol 2020;9(3):1518-1525. doi:10.21037/tau.2019.09.12
II Study of Ultrafocal Brachytherapy for Low- and Intermediate-risk Prostate Cancer. Int J Radiat Oncol Biol Phys 2018;102:903-11.

51. Valerio M, Stricker PD, Ahmed HU, et al. Initial assessment of safety and clinical feasibility of irreversible electroporation in the focal treatment of prostate cancer. Prostate Cancer Prostatic Dis 2014;17:343-7.

52. van den Bos $W$, de Bruin DM, Jurhill RR, et al. The correlation between the electrode configuration and histopathology of irreversible electroporation ablations in prostate cancer patients. World J Urol 2016;34:657-64.

53. Ting F, Tran M, Böhm M, et al. Focal irreversible electroporation for prostate cancer: functional outcomes and short-term oncological control. Prostate Cancer Prostatic Dis 2016;19:46.

54. Zlotta AR, Djavan B, Matos C, et al. Percutaneous transperineal radiofrequency ablation of prostate tumour: safety, feasibility and pathological effects on human prostate cancer. Br J Urol 1998;81:265-75.

55. Orczyk C, Brew-Graves C, Williams N, et al. Prostate radiofrequency ablation focal treatment (proRAFT): Results of a prospective development study for localised prostate cancer. Eur Urol Suppl 2018;17:e784-5.

56. Sivaraman A, Barret E. Focal Therapy for Prostate Cancer: An "A la Carte" Approach. Eur Urol 2016;69:973-5. 